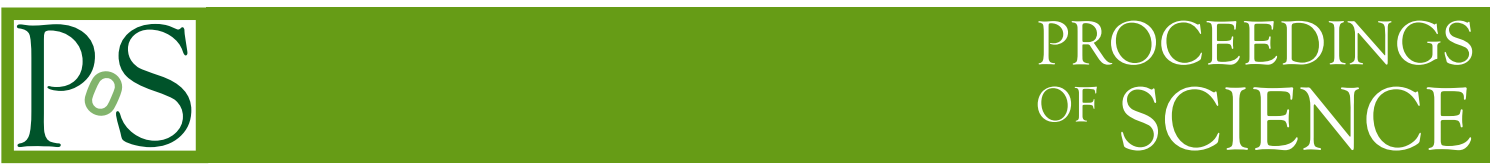

\title{
A database of charged cosmic rays (CRDB)
}

\section{David Maurin*}

LPSC, Université Grenoble-Alpes, CNRS/IN2P3, 53 avenue des Martyrs, 38026 Grenoble, France

E-mail: dmaurin@lpsc.in2p3.fr, crdatabase@lpsc.in2p3.fr

\section{Frédéric Melot}

LPSC, Université Grenoble-Alpes, CNRS/IN2P3, 53 avenue des Martyrs, 38026 Grenoble, France

\section{Richard Taillet}

LAPTh, UMR 5108 Université de Savoie - CNRS, BP 110, 74941, Annecy-le-Vieux, France

\begin{abstract}
We present an on-line database (http://lpsc.in2p3.fr/crdb) and associated on-line tools (data selection, data export, plots, etc.) for charged cosmic-ray (CR) measurements. The experimental setups (type, flight dates, techniques) from which the data originate are included in the database, along with the references to all relevant publications. Since its first release V1.1 (April 2013), the current CRDB version V2.1 (June 2014) includes new options and tools based on many user suggestions (additional plots, GALPROP export, REST interface, etc.). Other changes are part of our ongoing effort to improve this tool to the community (new structure for modulation values, corrections, etc.). We plan to include, among other improvements, new 'homogeneous' sets of solar modulation parameters (for all sub-experiments), $Z>30$ species, and higher energy CR data.
\end{abstract}

Cosmic Rays and the InterStellar Medium - CRISM 2014

24-27 June 2014

Montpellier, France

\footnotetext{
*Speaker.
} 


\section{Introduction}

Since the discovery of cosmic rays (CR) a century ago, instrumental capabilities have steadily improved. A large variety of types of experiments (balloon- or satellite-borne experiments, those flown on a shuttle, those installed on the international space station, or ground-based experiments) and techniques have been used (nuclear emulsions, drift chambers, Cerenkov counters, spectrometers, etc.) to refine our knowledge of the CR composition and spectrum.

In the last twenty years, a lot of effort has been devoted in measuring the CR composition at higher energy $(\gtrsim \mathrm{TeV})$. Very accurate isotopic data were also provided in the low energy range $\sim 100-500 \mathrm{MeV} / \mathrm{n}$ (Voyager 1\&2, Ulysses, and ACE), over an extended period of time. The recently installed AMS-02 experiment on the International Space Station has started to provide impressive measurements in the $\mathrm{GeV} / \mathrm{n}-\mathrm{TeV} / \mathrm{n}$ range (Aguilar et al. 2013). Interestingly, most of the even oldest CR measurements are not outdated yet. Indeed, many instruments are designed to focus on specific CR species: neither all instruments have the isotopic resolution capabilities nor have all species been measured repeatedly. Old experiments are also useful to inspect a possible chargesign dependence (22 year cycle) of the Solar modulation effect as a function of the Sun polarity (Potgieter 2013). These data can then be used in the context of Solar physics, Galactic CR propagation studies, and dark matter indirect detection studies, or for CR experimentalists who wish to compare their data to previously published ones.

A very important issue is to provide solar modulation levels for each dataset. In principle, a flight period for a given instrument is uniquely associated to a Solar activity period. For each sub-experiment of the database, a unique modulation level thus can be attached. In practice, its determination depends primarily on the choice of the (unknown) Solar modulation model. It also depends on the assumption made for the unknown interstellar (IS) flux and on the set of data used for its calculation. At least three different model-dependent approaches exist: the modulation potential given in the experimental papers is very often determined from an assumed ad-hoc local interstellar spectrum (LIS), which is modulated to match the data. Another widely used approach is to take a demodulated spectrum from another publication and modulate it to match the data. Another strategy is to rely on the worldwide network of neutron monitor data that gives an indirect estimation of the Solar modulation level (Usoskin et al. 2011). There is no consensus yet about which models and values to use, but some efforts are made in order to implement homogeneous sets of modulation values in CRDB.

The content of this proceeding is based on Maurin et al. (2013), where CRDB is described in details. A study of the uncertainties on time series of modulation level based on neutron monitor count rates can be found in Maurin et al. (2014): it is a starting point to provide new calculations of modulation levels for CRDB (Ghelfi et al., in prep.).

\section{Content of the database}

The CR database is based on a LAMP solution ${ }^{1}$. Authentication uses the https protocol to ensure a good level of confidentiality. (Only administrators use their own credentials to access

\footnotetext{
${ }^{1}$ The acronym LAMP refers to a stack of free open source softwares: Linux operating system, Apache HTTP server, MySQL database software, and PHP.
} 
protected areas.) All web pages are written using the PHP (Hypertext PreProcessor) language, with a global structure made in AJAX (Asynchronous JavaScript and XML). The third-party libraries jquery, jquery-ui, jquery.cluetip, and table-sorter are also used.

\subsection{Main fields in the MySQL structure}

The database engine is MySQL5, hosted at the Laboratoire de Physique and Cosmologie (LPSC) on a backed up server. Details about the structure, keys, tables, and links between the various elements can be found in Maurin et al. (2013). We only give below a brief description.

The CR data are connected to experiments, analyses, and publications. The first step for creating the database is to define what an experiment is. The need to define a sub-experiment arises because an experiment may consist of several detectors, or an instrument may have flown several times or over distinct periods. Data from a sub-experiment often involve several CR species, the analyses of which are published in one or several papers. Here are examples/definitions of keywords used in the database:

Experiment CREAM, AMS (name of the payload) or Balloon (YYYY) for unnamed balloons.

Sub-experiment Balloon (1972/07), CREAM-I (2004/12-2005/01), CREAM-II (2005/12-2006/01), Ulysses-HET (1990/10-1997/12).

Cosmic-ray quantity $\mathrm{C}$ (element), $1 \mathrm{H}$ (isotope), 1H-bar (antiproton), e- (lepton), or any ratio B/C, $10 \mathrm{Be} / \mathrm{Be}, \mathrm{e}+/(\mathrm{e}-+\mathrm{e}+)$, etc.

Energy axis Etot [GeV], R [GV], Ek [GeV], Ekn [GeV/n].

Data CR quantity measurements and uncertainties at one or several E bins.

Publication Journals or proceedings where the data were published.

\subsection{Cosmic ray data entry}

The structure of a CR data entry (energy, energy range, measurement and uncertainties) for any measured quantity is as follows:

$\langle\mathbf{E}\rangle$ 'Central' energy given in the publication in unit of 'Energy axis' (see above). If only the bin range (see below) is given in the publication, the geometric mean $\langle E\rangle=\sqrt{E_{\min } E_{\max }}$ is used.

Bin range Energy range, in the same unit as $\langle E\rangle$. If only $\langle E\rangle$ is provided, $E_{\min }=E_{\max }=\langle E\rangle$.

Value Measured CR quantity in $\left[\left(\langle E\rangle \mathrm{m}^{2} \mathrm{~s} \mathrm{sr}\right)^{-1}\right]$ for a flux, or unitless for a ratio.

Stat Err Statistical error (same unit as Value).

Syst Err Systematic error (same unit as Value); this is set to 0 if not given in publication. 


\subsection{Solar modulation level}

As there is no consensus yet on the solar modulation levels, we provide several sets of values (of the modulation parameters) and the underlying assumptions made for their determination. The two sets introduced in CRDB V2.1 are

1. From publication: this set is not homogeneous, since each value relies on IS fluxes and Solar modulation models (see below), which differ from one publication to another. We note that the underlying hypotheses are not always provided and that only a small fraction of the publications offers a modulation value at all.

2. NM [Uso11]: The modulation levels in this set are $\phi$ values in the Force-Field approximation, based on the NM data analysis of Usoskin et al. (2011). Monthly average values in the period July 1936 - December 2009 are interpolated for the flight dates of each sub-experiment. The ensemble of values forms a homogeneous set of $\phi$ for all sub-experiments, because each value is based on the same modelling.

As better constraints will be obtained on the IS flux and on CR data, new sets of values will be added in the future (Ghelfi et al., in prep.).

\section{Access to the database and tools}

The website ${ }^{2}$ is based on tabs, in which the user is guided by help boxes (identified by a 'question mark' icon). They are briefly described below, accompanied by two screenshots.

Welcome Quick description and organisation of the database, log of the latest changes, and link to download the database content in several formats.

ExPERIMENTS/DatA List of available data sorted by experiment names or dates (see Fig. 1).

Data eXtraction Main interface to retrieve data in ASCII files, ROOT ${ }^{3}$ macros and plots, and BiвTEX references for the selection (see Fig. 2).

AdmIN For authenticated users only: checks of the DB content and validation of submitted data.

Links Standard useful (here GCR-related) web links.

NEw DATA Interface to submit new data (appear in the DB after validation by authorised users).

This last tab allows anyone to interactively enter new data. This is an essential part of the database as it provides the community with the possibility to contribute to the completion of the database (either by adding data from new instruments, or adding missing data from older experiments). So far, only a couple of submissions were done, but we hope that this number will grow with time.

\footnotetext{
${ }^{2} \mathrm{http}: / / \mathrm{lpsc} . \mathrm{in} 2 \mathrm{p} 3 . \mathrm{fr} / \mathrm{crdb}$

${ }^{3}$ http://root.cern.ch
} 


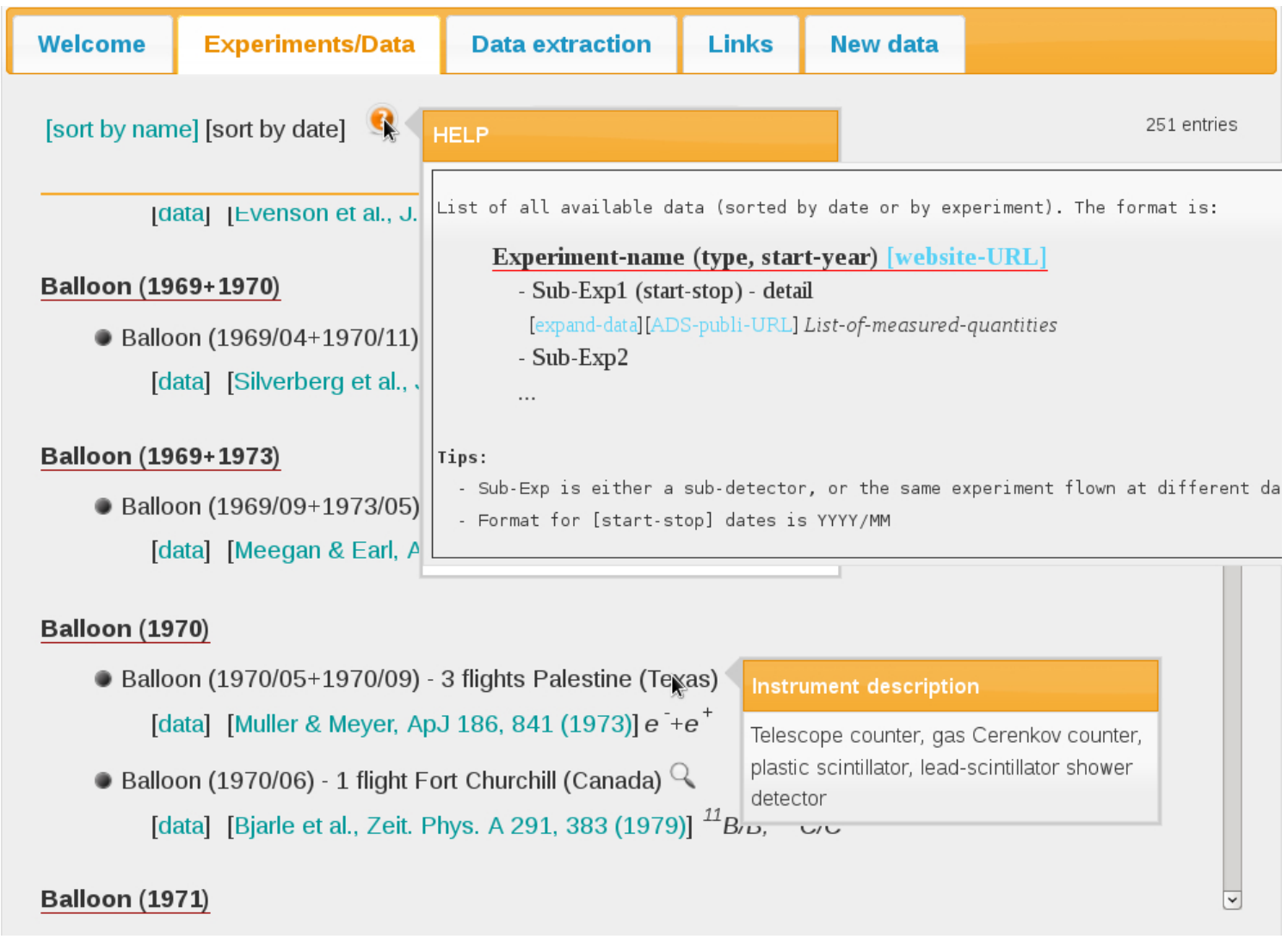

Figure 1: ExPERIMENTS/Data tab content. The help box is activated by clicking on the [question mark] icon and a picture of the instrumental setup (not shown) pops-up when clicking on the [magnifying glass] icon. The Instrument description box appears for a mouse-over action on the sub-experiment name. A click on [data] pops-up a new window with the data entries and a summary of all informations (not shown).

\section{Conclusion}

The cosmic-ray database (CRDB) includes $e^{-}, e^{+}, \bar{p}$, and nuclide data up to $Z=30$ for energies below a few $\mathrm{TeV} / \mathrm{n}$. Each $\mathrm{CR}$ data is linked to a description of the instrument that measured it (flight dates, picture of the experiment setup, techniques used, etc.) and to the ADS reference in which it was published: the first release contained more than 200 experiments and 200 publications, and this number is continuously growing. (We encourage experimentalists to submit their data once they become public.).

The data can be extracted according to a selection on the CR quantity, the energy range, the experiments, and the epoch of measurement: ASCII files, ROOT macros, plots, and BIBTEX for the corresponding publications are then readily available. Since CRDB V1.3, we have also implemented (on request) a REST interface ${ }^{4}$. The possibility to add new data by means of a user-friendly interface enables the database to be a collaborative tool for the CR community, provided enough people take an interest, use it, and help expand it.

\footnotetext{
${ }^{4}$ An example call using a command line in an xterm reads (click on [REST interface] in Fig. 2 for all parameters) wget 'http://lpsc.in2p3.fr/crdb/rest.php?num=C\&energy_type=EKN' - - output-document=file.
} 


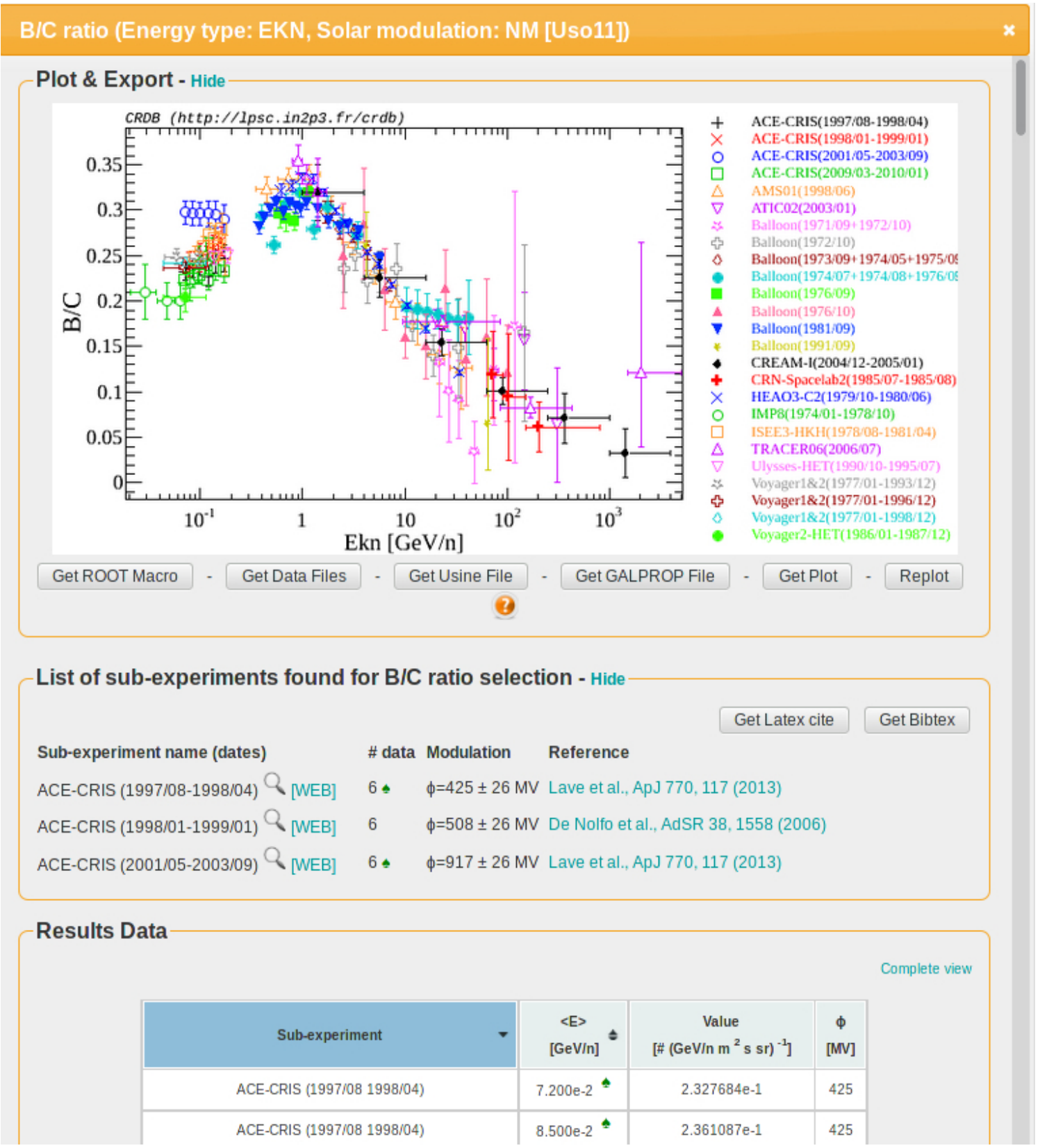

Figure 2: Snapshot of the result of the Data EXTRACtion operation. This pop-up window appears after the selection step (not shown). Buttons, links, and tables give access to raw data and plots.

The statistics of the website visits (set up since V1.2, October 2013), at the date of writing of this proceeding (August 2014), reveals that more than 1100 different IP addresses connected to CRDB, and more than 15000 pages/requests were made from many countries: Italy (19\%), USA (13.5\%), Germany (13\%), France/CERN (12.5\%), China (7\%), South Africa (6\%), Japan (6\%), Austria (4\%), Spain (2\%), and many other countries $\lesssim 1 \%$ each (UK, Finland, Sweden, Russia, India, Estonia, Netherlands, Portugal, Korea, etc.). Given these numbers, the CRDB initiative can be considered to be a success, the database and interface proving to be a very useful tool to the 
community. This encourages us to go further and to extend it, in the near future, to heavier species $(Z>30$ ), and to a larger energy domain (data from ground-based detectors $\gtrsim \mathrm{TeV} / \mathrm{n}$ data). Comments, questions, suggestions, and corrections can be addressed to crdatabase@lpsc.in2p3. fr.

Acknowledgements We thank L. Baldini, F. Barao, B. Coste, C. Deil, T. Delahaye F. Donato, M. Duranti, C. Evoli, F. Giovacchini, I. Grenier, S. Haino A. Karelin, M. Mosotho, A. Putze, A. Strong, L. Sujie, A. Vincent, for their positive feedback, help, and corrections/suggestions on CRDB. This work is part of the USINE project (CR propagation code). It has been financially supported by the PNHE.

\section{References}

M. Aguilar, G. Alberti, E. Alpat, et al., First Result from the Alpha Magnetic Spectrometer on the International Space Station: Precision Measurement of the Positron Fraction in Primary Cosmic Rays of 0.5-350 GeV, PRL 110, 141102 (2013).

D. Maurin, F. Melot, and R. Taillet, A database of charged cosmic rays, A\&A 569, 32 (2014) [arXiv:1302.5525].

D. Maurin, A. Cheminet, L. Derome, A. Ghelfi, and G. Hubert, Neutron monitors and muon detectors for solar modulation studies: Interstellar flux, yield function, and assessment of critical parameters in count rate calculations, AdSpR 55, 363 (2015) [arXiv:1403.1612].

M. Potgieter, Solar Modulation of Cosmic Rays, LRSP 10, 3 (2013) [arXiv:1306.4421].

I. G. Usoskin, G. A. Bazilevskaya, and G. A. Kovaltsov, Solar modulation parameter for cosmic rays since 1936 reconstructed from ground-based neutron monitors and ionization chambers, JGR 116, 2104 (2011). 